Review

\title{
Cortical activity during walking and balance tasks in older adults and in people with Parkinson's disease: A structured review
}

\author{
Samuel Stuart ${ }^{\mathrm{a}}$, Rodrigo Vitorio ${ }^{\mathrm{b}}$, Rosie Morris ${ }^{\mathrm{a}}$, Douglas N. Martini ${ }^{\mathrm{a}}$, Peter C. Fino ${ }^{\mathrm{a}}$, \\ Martina Mancini ${ }^{\mathrm{a} \text {,* }}$ \\ a Oregon Health \& Science University, Department of Neurology, Portland, OR, USA \\ ${ }^{\mathrm{b}}$ Universidade Estadual Paulista (UNESP), Instituto de Biociências, Campus Rio Claro, Brazil
}

\section{A R T I C L E I N F O}

\section{Keywords:}

Functional near infrared spectroscopy (fNIRS)

Electroencephalography (EEG)

Cortical activity

Walking

Balance

Older adults

Parkinson's disease

\begin{abstract}
A B S T R A C T
An emerging body of literature has examined cortical activity during walking and balance tasks in older adults and in people with Parkinson's disease, specifically using functional near infrared spectroscopy (fNIRS) or electroencephalography (EEG). This review provides an overview of this developing area, and examines the disease-specific mechanisms underlying walking or balance deficits. Medline, PubMed, PsychInfo and Scopus databases were searched. Articles that described cortical activity during walking and balance tasks in older adults and in those with PD were screened by the reviewers. Thirty-seven full-text articles were included for review, following an initial yield of 566 studies. This review summarizes study findings, where increased cortical activity appears to be required for older adults and further for participants with PD to perform walking and balance tasks, but specific activation patterns vary with the demands of the particular task. Studies attributed cortical activation to compensatory mechanisms for underlying age- or PD-related deficits in automatic movement control. However, a lack of standardization within the reviewed studies was evident from the wide range of study protocols, instruments, regions of interest, outcomes and interpretation of outcomes that were reported. Unstandardized data collection, processing and reporting limited the clinical relevance and interpretation of study findings. Future work to standardize approaches to the measurement of cortical activity during walking and balance tasks in older adults and people with PD with fNIRS and EEG systems is needed, which will allow direct comparison of results and ensure robust data collection/reporting. Based on the reviewed articles we provide clinical and future research recommendations.
\end{abstract}

\section{Introduction}

Parkinson's disease (PD) causes walking and balance deficits $[1,2]$ that lead to increased falls, reduced mobility and quality of life [3]. In general, $60 \%$ of older adults ( $>$ 80years old) have gait disorders [4] which cause $17 \%$ of falls [5], with higher incidences in PD [6]. While some gait impairments in $\mathrm{PD}$, such as slow gait, may relate to primary pathophysiology (i.e. bradykinesia), others, such as increased gait variability may be compensatory in nature [7]. Animal model evidence denotes that voluntary movements are derived from motor commands projecting from the cortex to the brainstem and spinal cord [8,9-12]. Goal-directed behaviors, such as walking, are always accompanied by automatic processes of postural control involving balance adjustment and muscle tone regulation [8] that rely more on subcortical structures (i.e. basal ganglia and brain stem) [8]. PD impacts subcortical pathways leading to dysfunctional automatic movement control, which is suggested to be accompanied by a compensatory shift to more voluntary cortical control $[10,13,14]$. Therefore, walking may rely heavily on compensation from cortical structures in PD, which may also increase motor performance variability.

In parallel, other studies focus on the role of cognition in balance and gait dysfunction in PD [15]. Extensive associative behavioral studies have linked cognition to walking and balance performance $[16,17]$, however these previous experiments have not investigated the neural mechanisms involved. Changes in brain structure and connectivity with ageing and PD impact cognitive processes, walking and balance $[18,19]$, likely due to involvement of common neural centers. Specifically, white matter structural changes, lower hippocampal and anterior cortex volume relate to executive-attentional deficits with links to walking and balance impairment [15,20-22]. Therefore, diseasespecific impairments of brain activity, motor control and cognition potentially mediate task performance. Examining underlying cortical

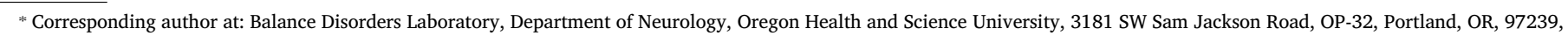
USA.

E-mail address: mancinim@ohsu.edu (M. Mancini). 
activity involved in walking and balance in older adults and PD will allow further clarification of disease-specific links between these features.

Traditionally, brain structure and function have been studied using imaging techniques, such as functional magnetic resonance imaging $[18,23,24]$. The majority of studies have focused on investigation of motor regions (such as supplementary motor area (SMA), premotor (PMC), primary motor (M1) and sensorimotor cortices (SMC)) [25], with only a few recent studies examining the involvement of cognitive regions (such as prefrontal cortex (PFC)) [26-28]. Unfortunately, imaging results are limited as the head has to remain still and assays of walking and balance are used to mimic task performance (e.g. virtual reality or mental imagery), or studies use simple, single-segment motor tasks (e.g. finger tapping or button pressing) to infer cortical or subcortical activity related to general motor control.

Recent technological advances have allowed investigation of cortical activity during real-time walking or balance tasks in older adults and PD using functional near infra-red spectroscopy (fNIRS) or electroencephalography (EEG). However, this emerging body of literature has yet to be compiled for comprehensive methodological evaluation and interpretation. Greater understanding of cortical activity involved in walking and balance with age and PD will allow PD-specific cortical targets for intervention development to be uncovered. Therefore, we focused this review on the following: 1) cortical activity during realtime walking and balance tasks in older adult and PD subjects; 2) study protocols including cortical activity instrumentation and outcomes; and 3) clinical and future research recommendations.

\section{Methods}

\subsection{Search strategy}

Key search terms and synonyms are displayed within Fig. 1. All key terms were matched and exploded with medical subject headings (MeSH). Databases searched included Medline and PsychInfo, Scopus and PubMed (Fig. 2). Studies were deemed relevant if they incorporated terminology that focused on cortical activity during a walking or balance task in older adults or PD in the title, abstract or keywords. Initial title screen for relevant articles was performed by the reviewer (SS). Titles and abstracts were then further screened by three reviewers (SS; $\mathrm{RV} ; \mathrm{RM})$. A final full-text review was performed if further clarity was required for articles meeting review criteria (Table 1).

\subsection{Data extraction}

Data was extracted and synthesized into tables by the reviewer (SS) and confirmed by the other reviewers (RV, RM, DM, PF) (Tables 2 and 3). Extracted data included first author and year of publication, population characteristics, cohorts, task, type of measurement device, regions of interest (ROI), signal pre-processing, outcome measures, key findings and interpretation.

\section{Results}

\subsection{The evidence base}

The initial search yielded 556 articles following duplicate exclusion (Fig. 2) [29]. Initial title and abstract screening resulted in 56 articles of interest of which 37 were identified for inclusion by three reviewers (SS, RV, RM), with consensus of article inclusions following consultation with the other adjudicating reviewers (DM, PF, MM). Reasons for exclusion of studies are included as Supplementary Material 1.

\subsection{Participants}

The reviewed articles $(n=37)$ investigated older adults and people with PD with an average age of 66.5 years and 78.2 years, respectively. Both male and female participants were recruited to the majority of studies, although several did not report gender characteristics (Table 2). Generally, PD participants were tested "ON" their parkinsonian medications, although some studies did not report medication status $[30,31]$. Fewer studies examined PD compared to older adult subjects.

\section{Search string key terms}

Older adult or neurodegenerative group: "Older adult" OR "healthy older" OR elderly OR "Parkinson*" (TITLE-ABS-KEY)

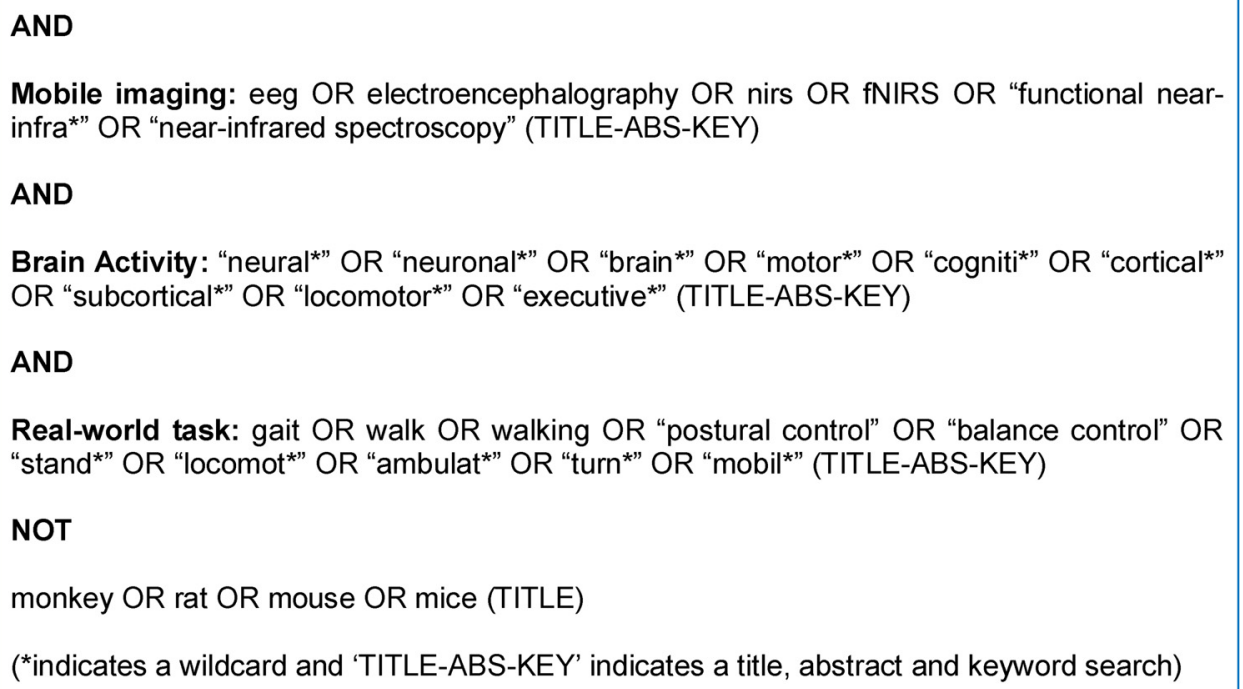

Fig 1. Search string used for study acquisition. This illustrates the four key terms used for this review and the synonyms used for each. 


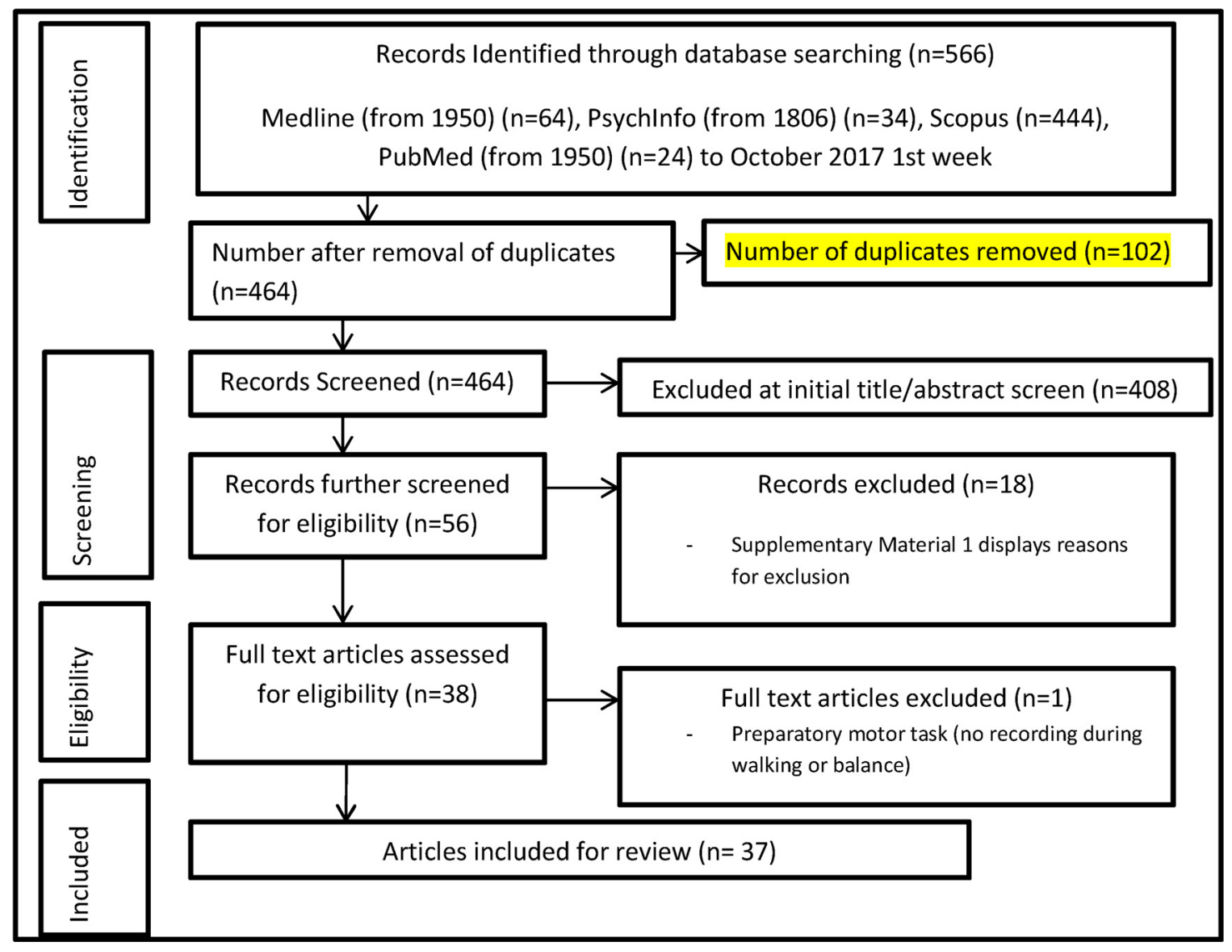

Fig. 2. PRISMA flow chart of study design. This illustrates the yield of the search strategy at each stage of the study selection process.

Table 1

Article Inclusion and Exclusion Criteria.

\begin{tabular}{|c|c|}
\hline Inclusion Criteria & Exclusion Criteria \\
\hline $\begin{array}{l}\text { - Studies were included if they reported cortical activity during a walking or balance } \\
\text { task (such as; walking, turning, obstacle crossing, standing, standing with } \\
\text { perturbation etc.) } \\
\text { - Whereby articles included another clinical cohort (e.g. multiple Sclerosis) or a static } \\
\text { imaging task, only data pertaining to older adult or PD participants during a } \\
\text { walking or balance task was reviewed. }\end{array}$ & $\begin{array}{l}\text { - Articles were excluded if they involved simple motor tasks (i.e. button pressing, } \\
\text { finger tapping/movement, wrist/elbow extension or flexion, a single step) as these } \\
\text { were not considered to be walking or balance tasks. } \\
\text { - Only articles written in English were considered and abstracts, case studies, reviews, } \\
\text { commentaries, discussion papers, editorials or conference proceedings were excluded. } \\
\text { - Experiments that involved assays of walking or balance (e.g. motor imagery or virtual } \\
\text { reality) without task performance, or that involved pre and post-task cortical } \\
\text { measurement rather than during task recording were also excluded, as these may not } \\
\text { represent activity that actually occurs during the task. } \\
\text { - Articles related to animal models (monkey, rat or mouse) were excluded with } \\
\text { separate key terms. }\end{array}$ \\
\hline
\end{tabular}

\subsection{Study protocols}

\subsubsection{Tasks}

Table 2 demonstrates that there was little consensus regarding the walking and balance tasks used within the reviewed studies. Studies primarily involved assessment of cortical activity during walking (normal walking; $\mathrm{n}=8$ treadmill, $\mathrm{n}=22$ over-ground) with fewer studies examining balance tasks ( $n=8$ quiet standing, $n=4$ with floor translation perturbations). The most common manipulation was a dualtask (cognitive or motor) $(\mathrm{n}=23)$, with other complex walking tasks also used such as different walking speeds $(n=2)$, obstacle crossing/ precision stepping $(n=5)$ or turning tasks $(n=2)$. Cognitive dualtasks consisted of a variety of speaking tasks, with reciting alternative letters of the alphabet most used $(n=10)$. Three studies used motor dual-tasks that involved carrying items or pressing buttons.

\subsubsection{Cortical activity monitoring instruments}

Table 2 shows that within the reviewed articles various fNIRS ( $\mathrm{n}=26$ studies) $[12,31-56]$ and EEG $(\mathrm{n}=11$ studies) $[30,57-66]$ instruments were used, which differed in terms of type/model, sampling frequency and number of channels. Different models of fNIRS devices emitted either LED or laser light, with inter-optode distances that ranged from $2.2 \mathrm{~cm}$ to $4 \mathrm{~cm}$. Similarly, different fNIRS and EEG devices used a range of materials to hold channels in place, such as semi-rigid plastic forms or neoprene head-bands/caps. The sampling frequency of the fNIRS devices varied from 1 to $10 \mathrm{~Hz}$, whereas EEG systems had frequencies that varied from 250 to $1024 \mathrm{~Hz}$. The number of channels also varied considerably between studies, with fNIRS systems ranging from 2 to 16 channels and EEG systems having a larger range of 3-72 channels. 


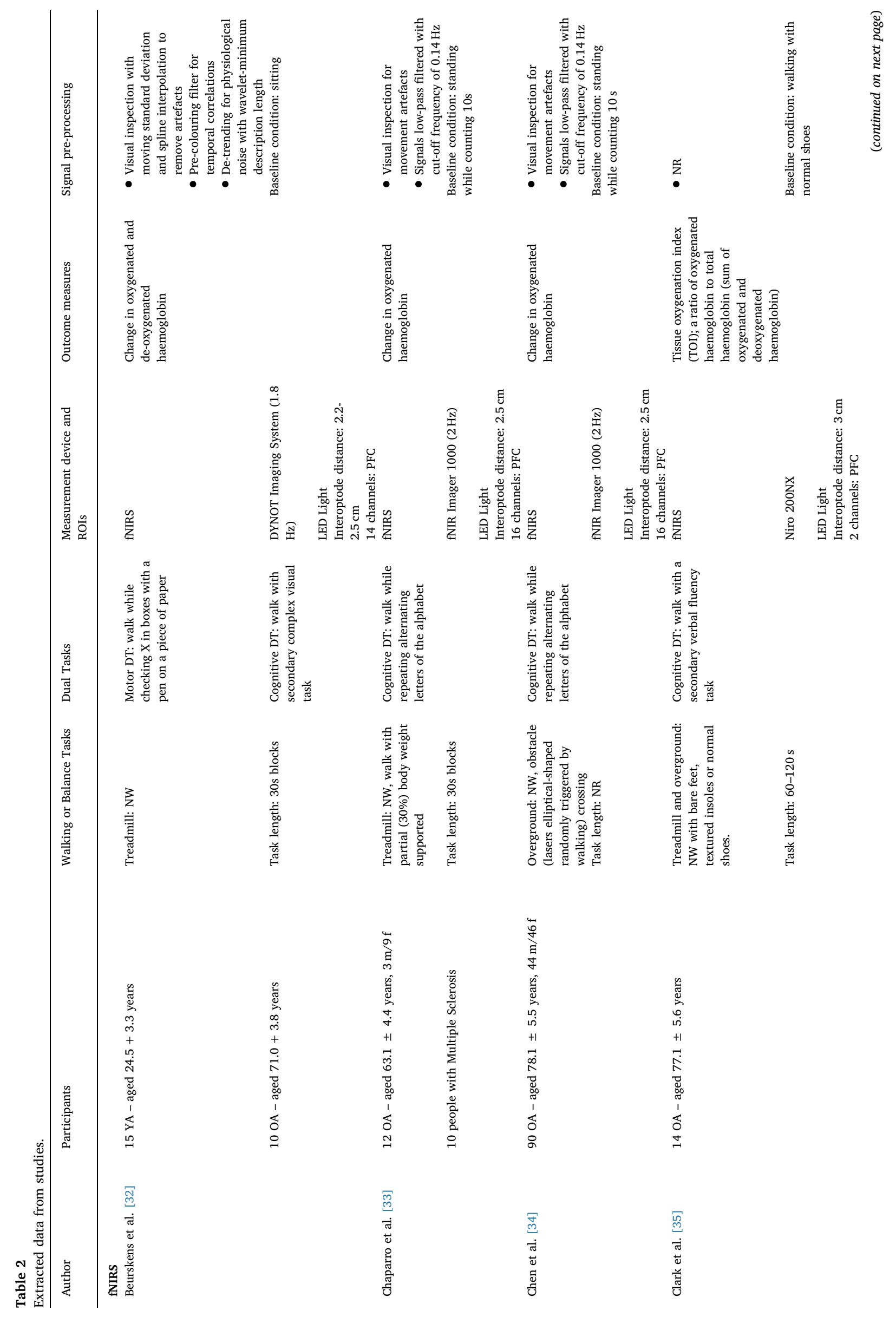




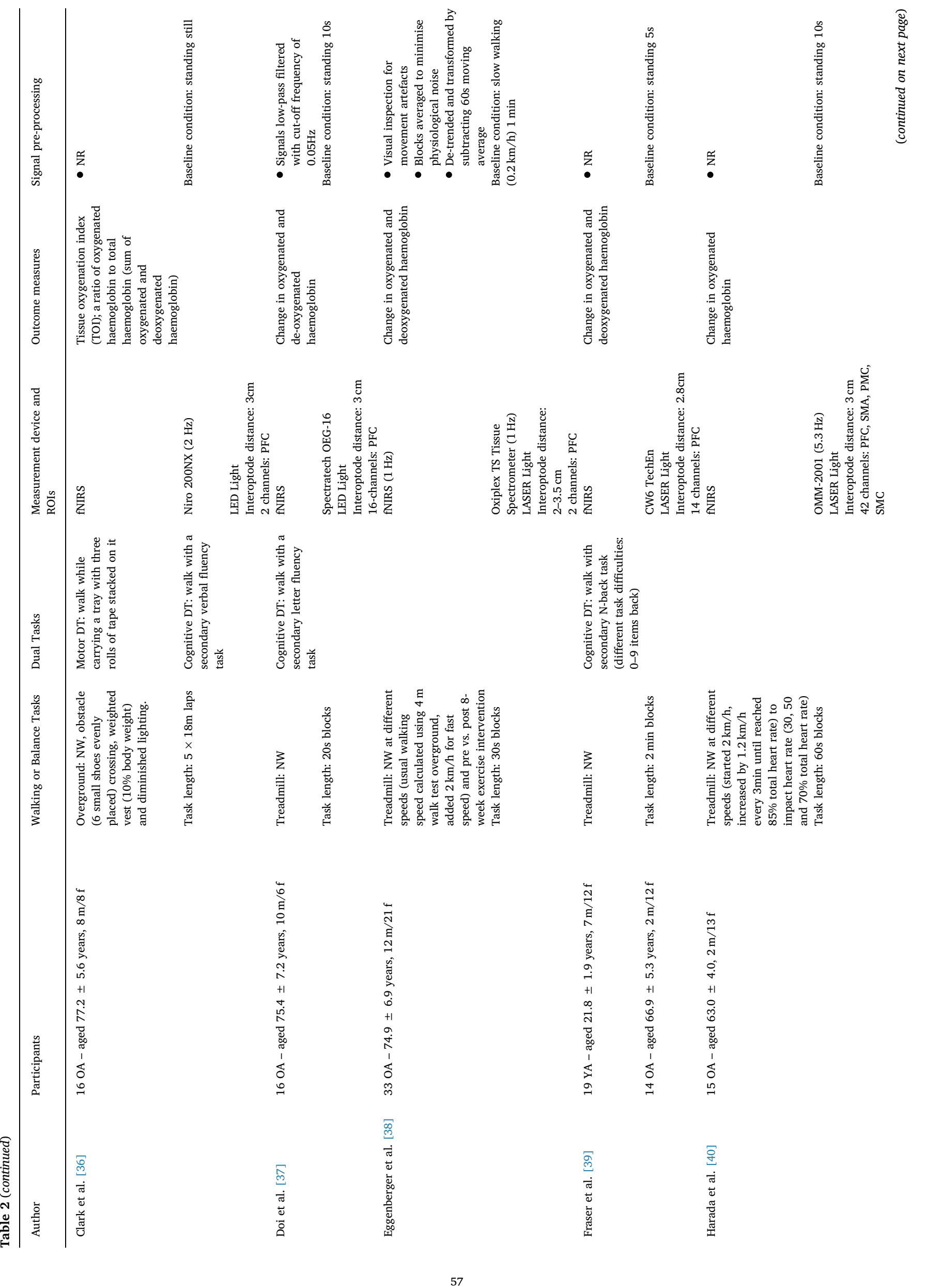




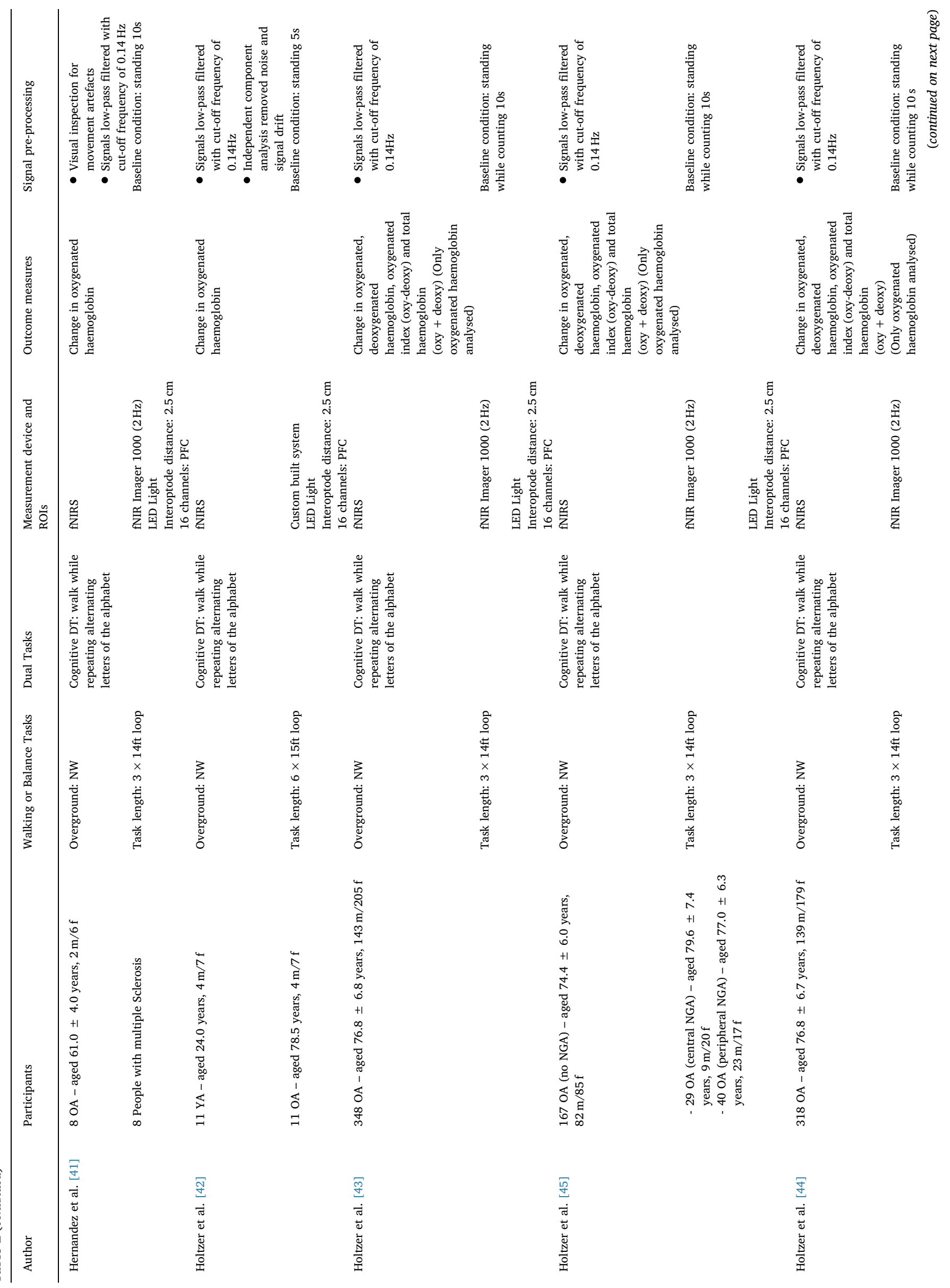




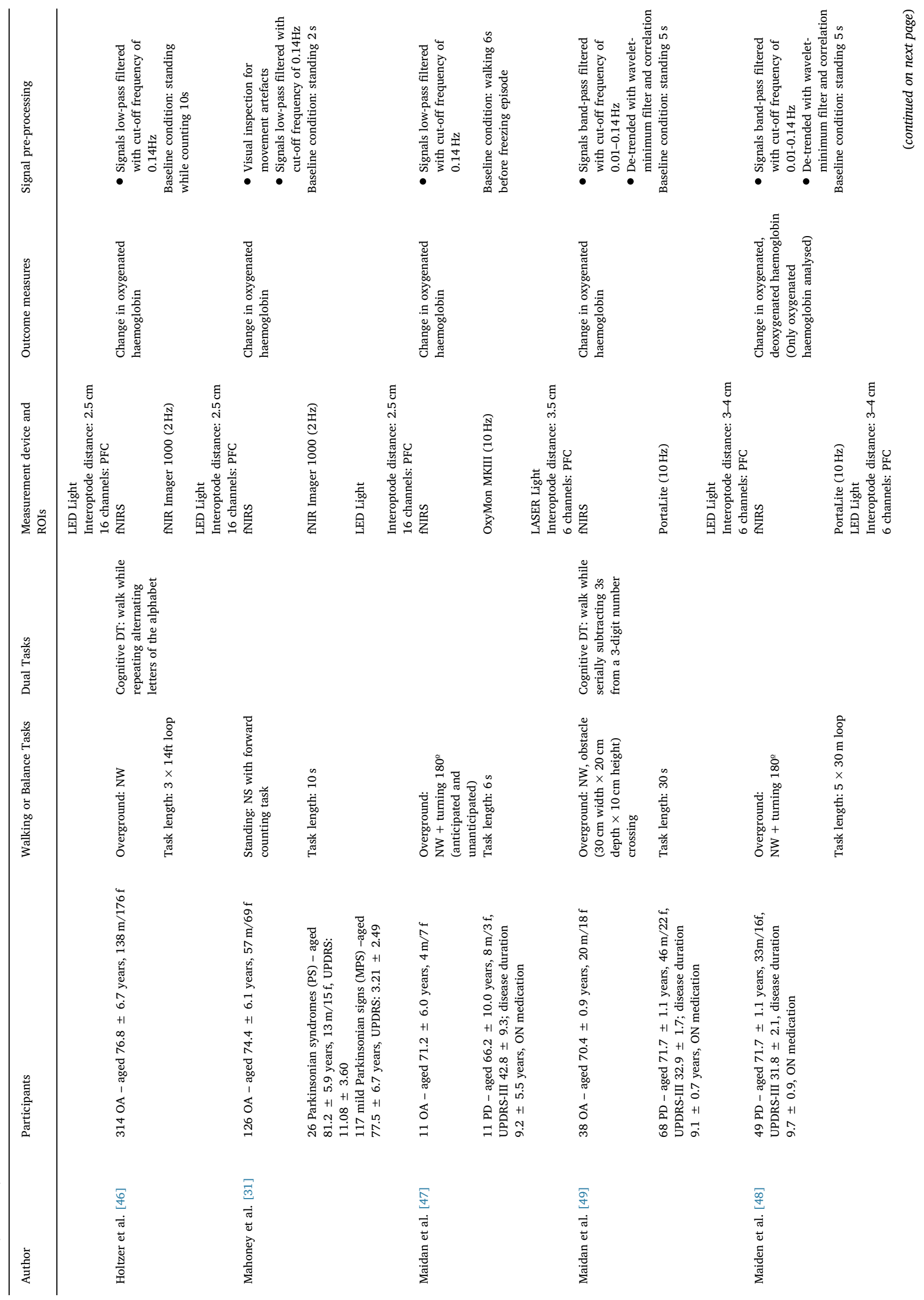




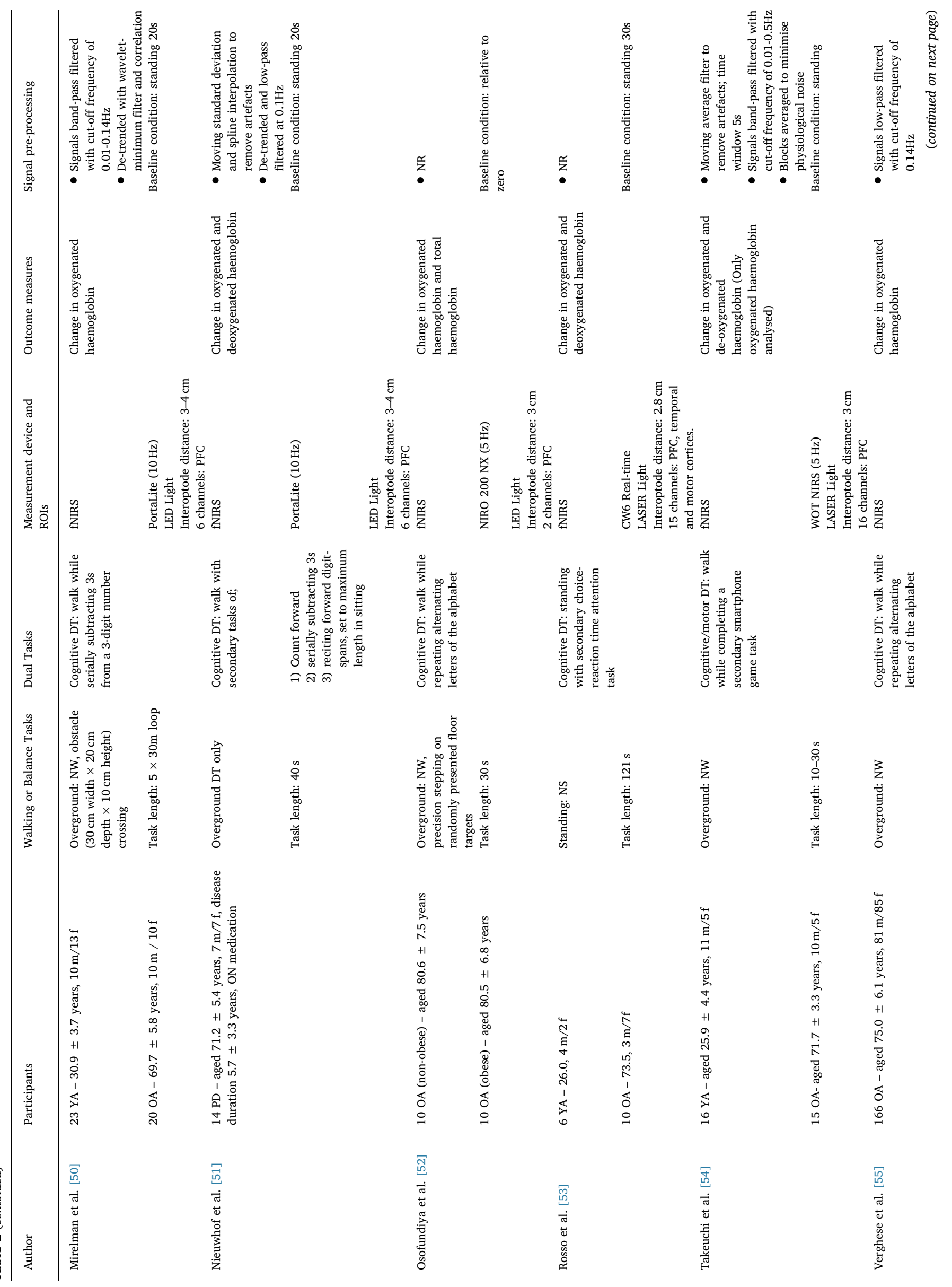




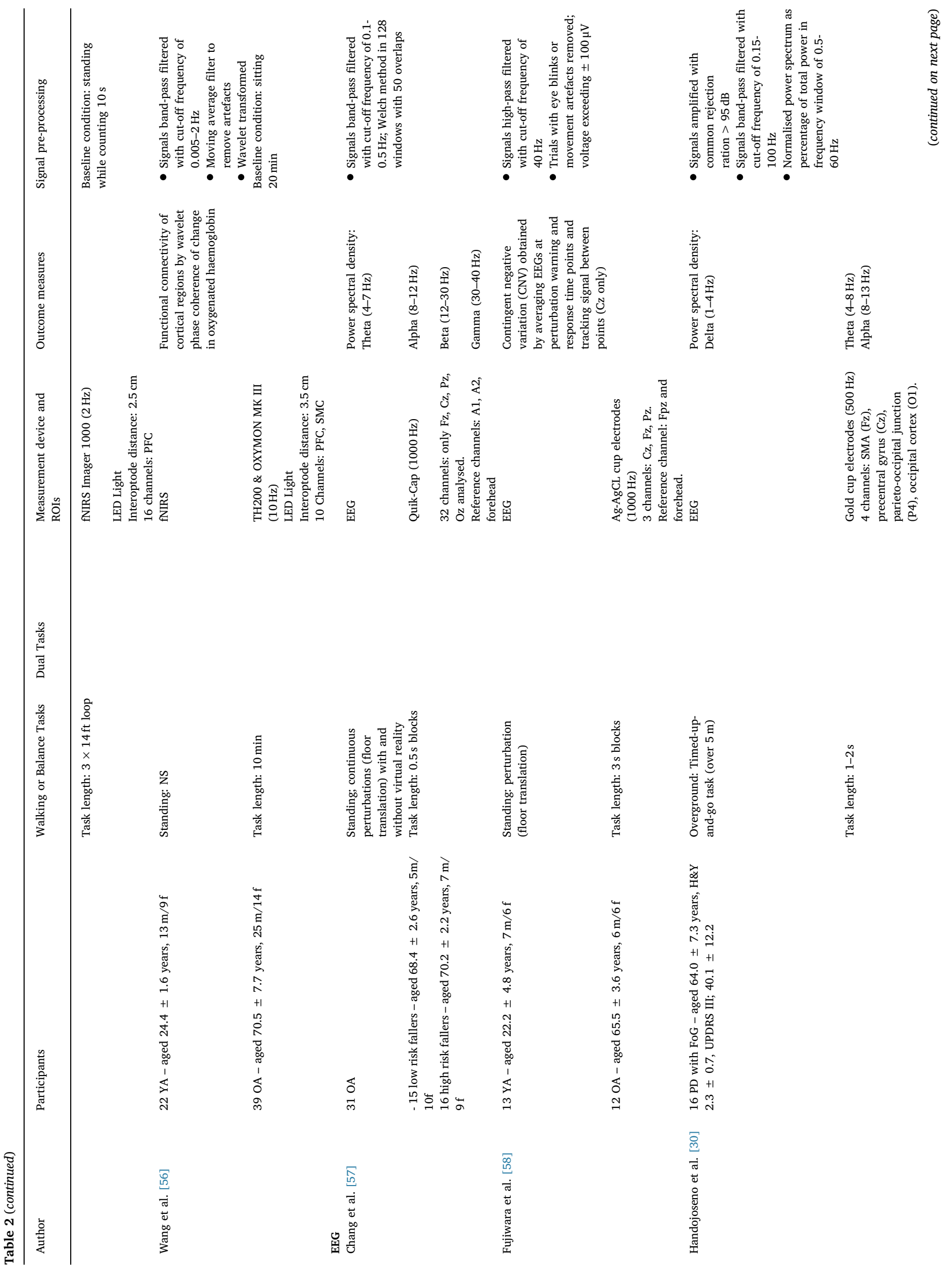




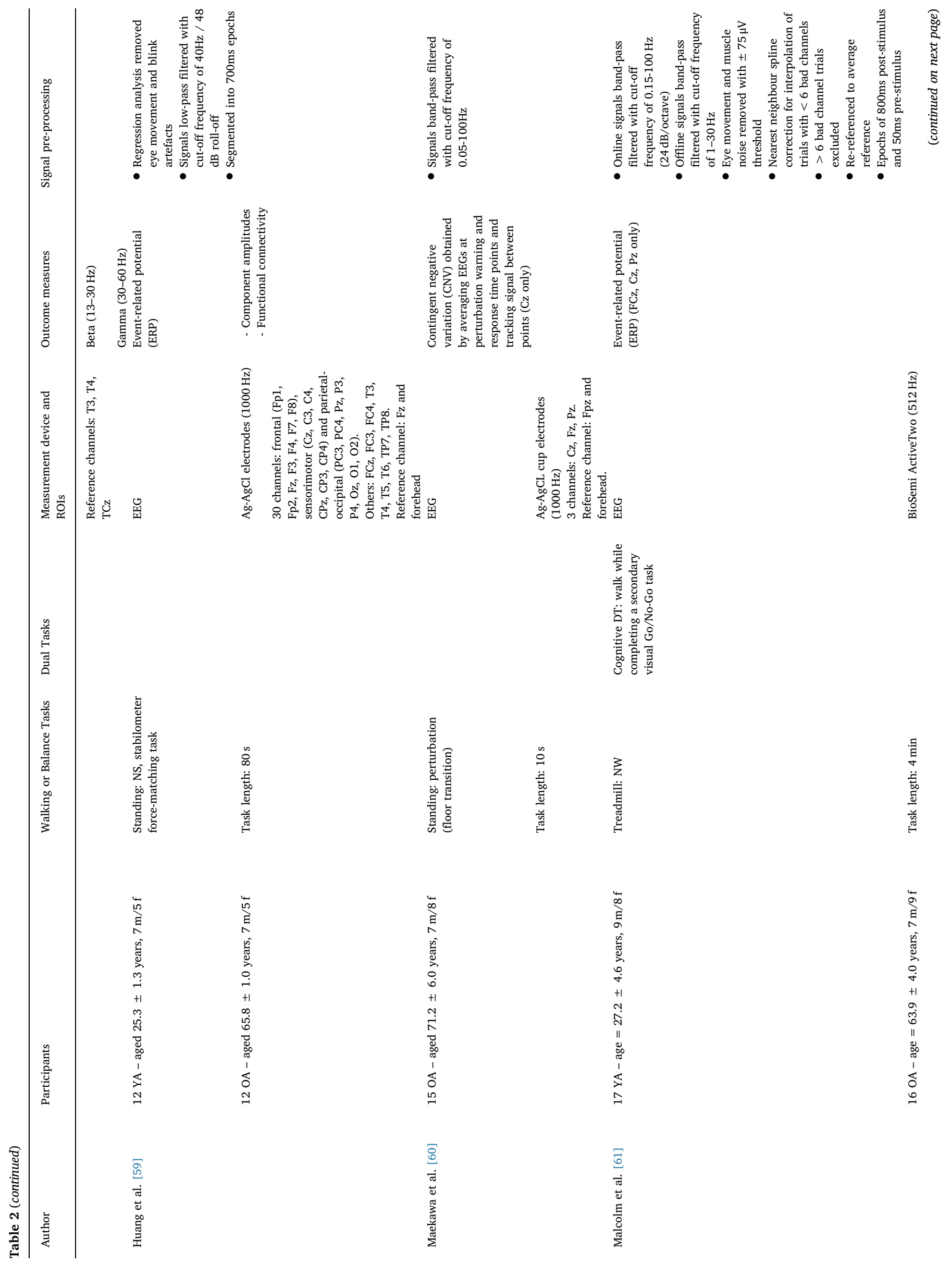




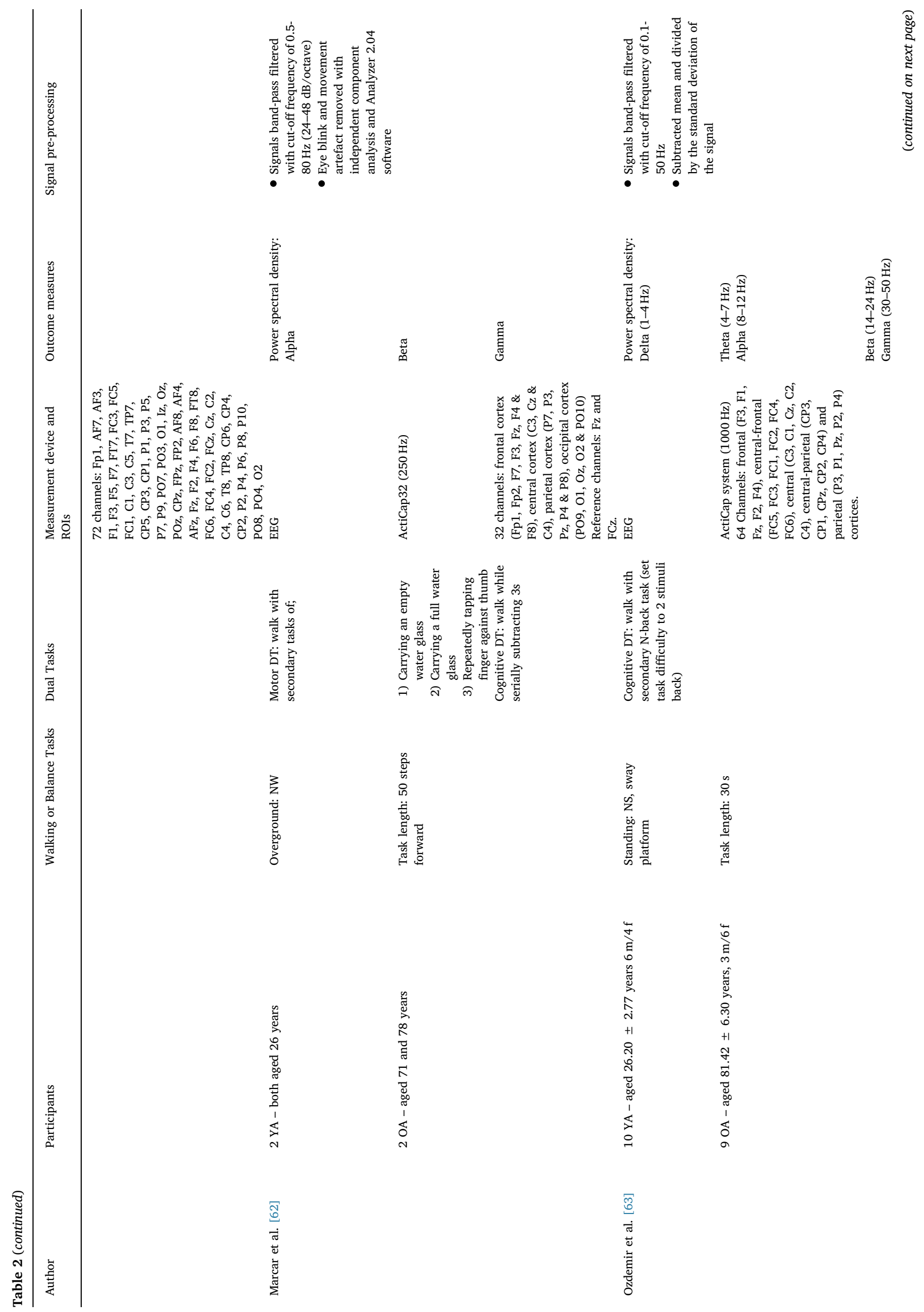




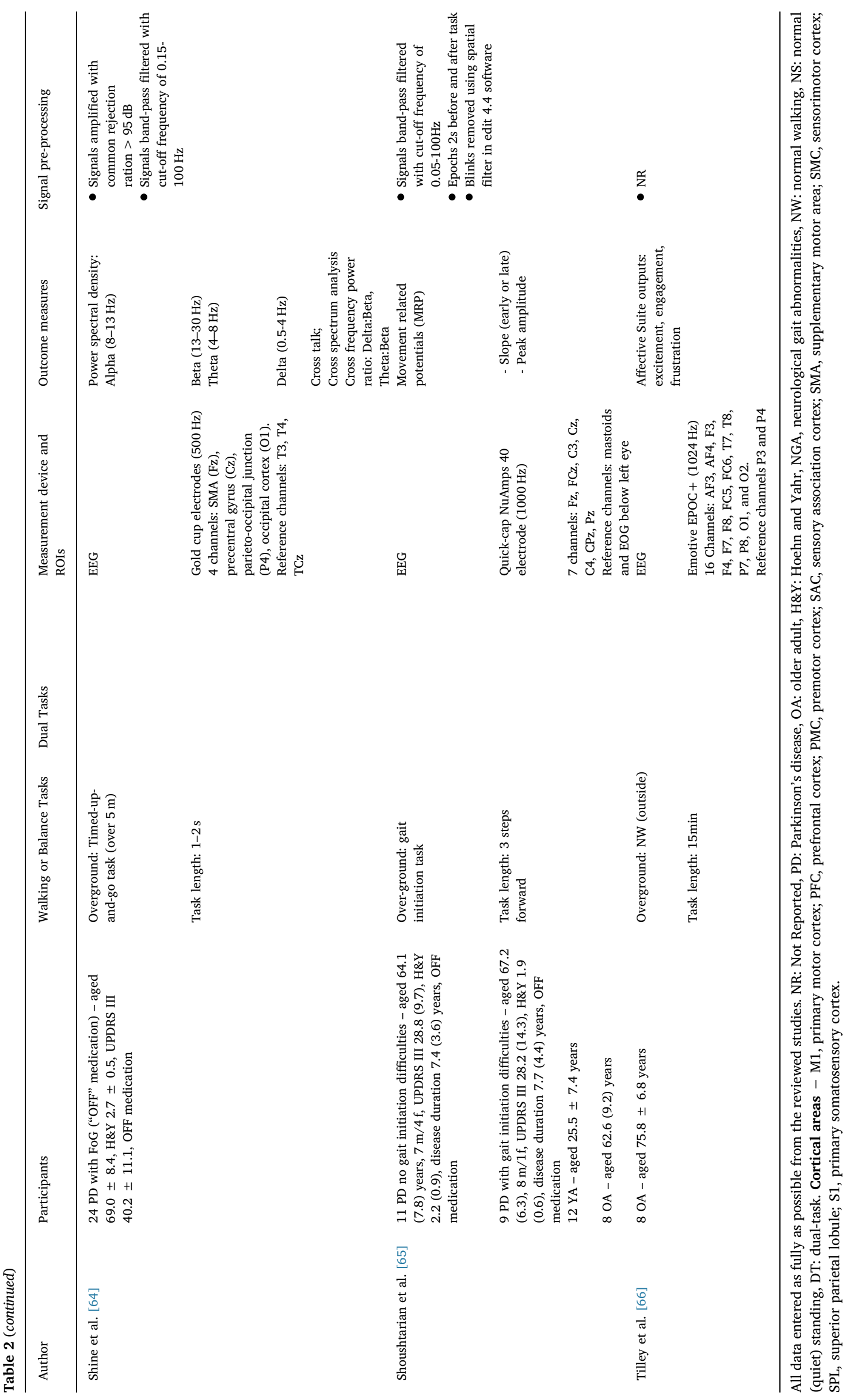




\subsection{Regions of interest}

All fNIRS studies examined the PFC, with only two studies also examining other ROIs (e.g. SMA, PMC, SMC). The EEG studies reported data from a range of different ROIs. For example, frontal, parietal, occipital, central and sensorimotor cortices, although not all of the reviewed studies described their results in terms of ROIs (i.e. just reported channels) and several studies combined ROIs (Table 2). Studies also varied in terms of the number of channels devoted to each ROI, for example one study [59] used multiple channels to denote ROIs whereas others used a single channel [30]. Several studies also used the same EEG channels but attributed recordings to different ROIs, e.g. $\mathrm{Cz}$ channel was used to report SMC, frontal lobe, central cortex and precentral gyrus.

\subsection{Cortical activity pre-processing and outcome measures}

Cortical activity pre-processing and outcome reporting was inconsistent (Table 2). Pre-processing for fNIRS involved visual signal inspection, filtering (low or band-pass) with de-trending or moving averages, and averaging across trial blocks. In contrast to previous research, none of the reviewed fNIRS studies used reference channels (inter-optode distance $<1.5 \mathrm{~cm}$ ) to account for peripheral tissue signals.

Twenty-three fNIRS studies reported oxygenated haemoglobin relative to static baseline conditions ( $2 \mathrm{~s}-20 \mathrm{~min}$ duration), with three studies using dynamic baselines and few reporting deoxygenated haemoglobin or absolute values (i.e. total haemoglobin or total oxygenation index) (Table 2). One fNIRS study reported functional connectivity of ROIs [56]. Outcomes were primarily calculated from averages over task blocks ( $1 \mathrm{~s}$ to $2 \mathrm{~min}$ duration), which led to primarily intermittent walking or standing bouts being studied.

Table 3 shows that the pre-processing for EEG involved are range of techniques. For example, filtering (cut-offs ranged from 0.05 to $100 \mathrm{~Hz}$ ), regression, independent component analysis (ICA), voltage threshold cut-offs (e.g. $\pm 75 \mu \mathrm{V}$ ) or spatial filtering to remove blinks or movement artefacts and creating timing epochs for trial periods (e.g. $2 \mathrm{~s}$ windows). EEG studies used reference channels at the forehead, around the eye and peripheral regions to remove muscle, eye movement or other physiological signals.

Five of EEG studies reported power spectrum densities (PSD), with few studies reporting cross talk variables, functional connectivity, component amplitudes, event or movement-related potentials (MRP) or contingent negative variation (Table 2). One EEG study used the manufacturer software to obtain generic variables of "excitement", "engagement" and "frustration" [66]. PSD bandwidths for Alpha, Beta and Gamma were most commonly used ( $\mathrm{n}=5$ studies), with theta and delta reported in only three studies. However, between studies bandwidth thresholds varied (i.e. Alpha $=8-13 \mathrm{~Hz}$ [64] vs Alpha $=8-12 \mathrm{~Hz}$ [63]).

\subsection{Interpretation of cortical activity outcome measures}

Table 3 and Fig. 3 detail influence of age and PD on cortical activity outcomes during walking and balance tasks. Control groups, when included, were young adults or otherwise healthy elderly.

\subsubsection{Walking tasks}

Walking at comfortable speed generally increased cortical activity in both older adults and PD compared to control groups (young or older adults, respectively) or baseline measures (standing or sitting), with greatest activation in PD. Specifically, walking led to greater activation of frontal ROIs or networks [61,66], as well as the PFC $[40,42-44,47,48,50]$, SMA, PMC and SMC [40]. Although, after initial task exposure, activation levels decreased and tended to return to the baseline in older adults [43]. However, two reviewed studies reported that PFC activation was not increased during walking at comfortable speed in older adults $[32,42]$ compared to baseline (sitting/standing), and another study demonstrated reduced cortical activity in older adults and PD within the first three steps of walking [65] compared to baseline (standing). In addition, only one study also showed that, following an exercise intervention, PFC activity during walking reduced in older adults compared to before exercise [38].

Dual-task walking generally increased PFC activation compared to baseline (standing or sitting) and single-task walking in both older adults and PD, with greater activation in PD (Table 3). However, three studies reported no increase in cortical activity in older adults when walking under dual-task conditions compared to single-task [32,49,54]. Increased PFC activity with dual-task also predicted falls in older adults [55].

During complex walking conditions, such as obstacle crossing, turning, precision stepping and timed-up-and-go (TUG), cortical activation appeared task-specific. Specifically, cortical activity increased in primarily frontal and central ROIs prior to freezing episodes compared to walking during TUG in people with PD $[30,64]$. Turning had no effect of PFC activity in older adults, but reduced PFC activity in PD and increased activity in those who experienced freezing during turns $[47,48]$. Obstacle crossing and precision stepping increased PFC activity in older adults $[34,50,52]$. Cortical activity also increased during treadmill walking compared to over-ground walking [35].

\subsubsection{Balance tasks}

Quiet standing generally increased cortical activity in older adults and PD, shown by greater PFC activation compared to sitting [31] and greater functional connectivity of various ROIs compared to controls $[56,59]$. However, one study also reported reduced functional connectivity in standing compared to sitting in older adults [56]. Dual-task standing further increased PFC and temporal cortex activity in older adults [53]. Balance under perturbation (floor translation) increased cortical activity compared to normal standing in older adults $[60,63]$, but activation reduced over time with further perturbation exposure [60].

\section{Discussion}

This structured review summarized current literature on cortical activity during walking and balance tasks in older adults and PD, with a focus on real-time recording of fNIRS and EEG signals. The reviewed studies varied in terms of protocol, ROIs, outcomes and interpretation, with a lack of standardization throughout. Despite this, studies reported clinically relevant information regarding cortical mechanisms underlying mobility in older adults and PD.

\subsection{Study protocols}

Cortical activity results obtained from small cohorts that use various walking or balance tasks may not generalize to larger cohorts and limit the statistical power of the findings. This was evident within the reviewed studies, as only two PD-related studies had large sample sizes ( $n>30$ ) and a range of different walking and balance tasks were used (i.e. treadmill or over-ground walking, timed-up-and-go, turning, gait initiation, perturbations etc.), which led to some inconsistent findings. For example, in opposition to larger studies that used simple overground continuous walking tasks, those that studied more complex intermittent walking tasks (i.e. treadmill walking or turning) with small sample sizes reported no change or decreased cortical activity $[32,49]$. Larger cohort findings may be more robust, particularly for fNIRS results due to physiological variations between individuals [67], indicating that technology may be a limiting factor.

Most reviewed studies involved fNIRS with few using EEG, particularly with PD participants. A mobility-device trade-off appeared, whereby EEG was primarily used for static or controlled tasks (i.e. 


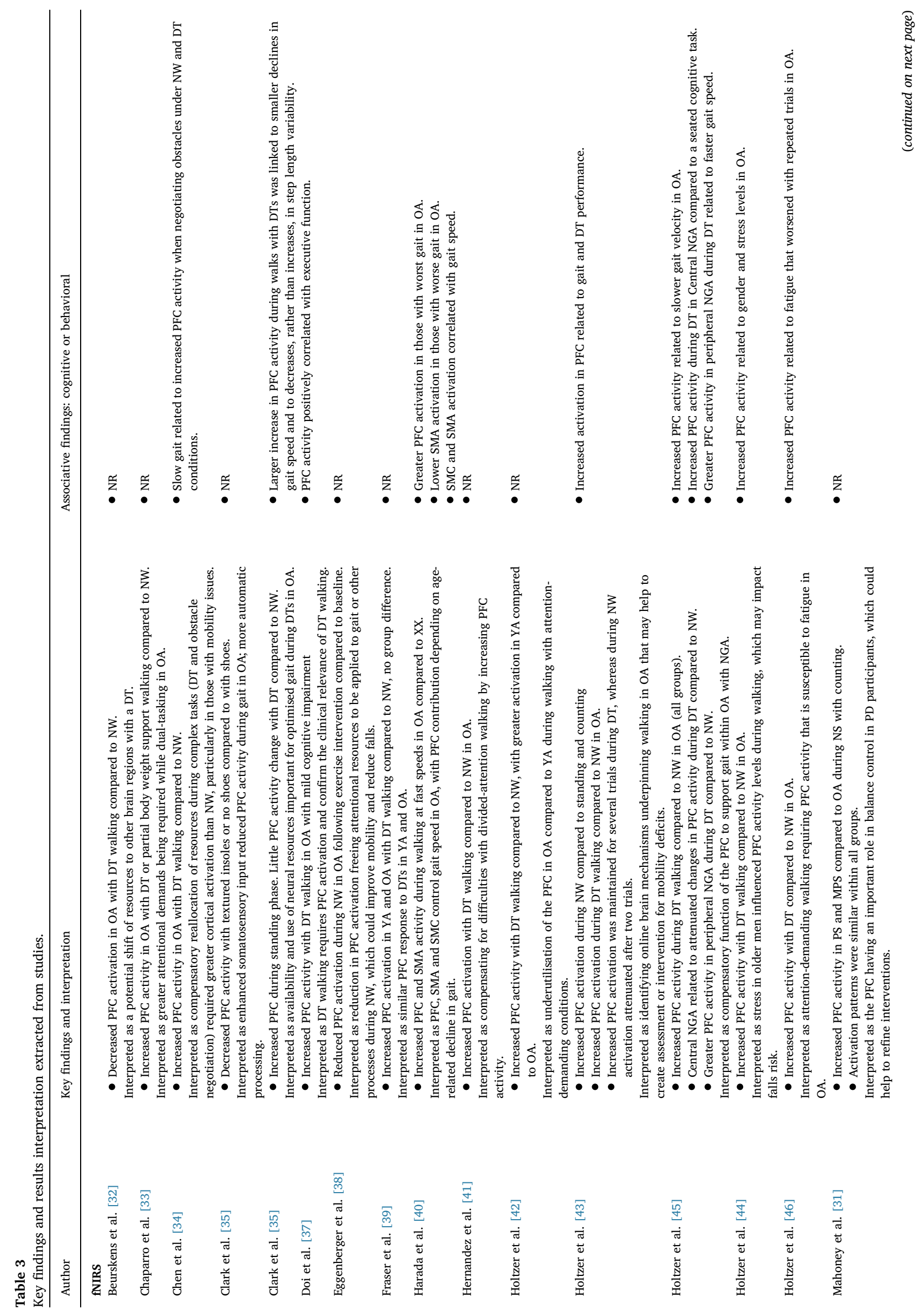




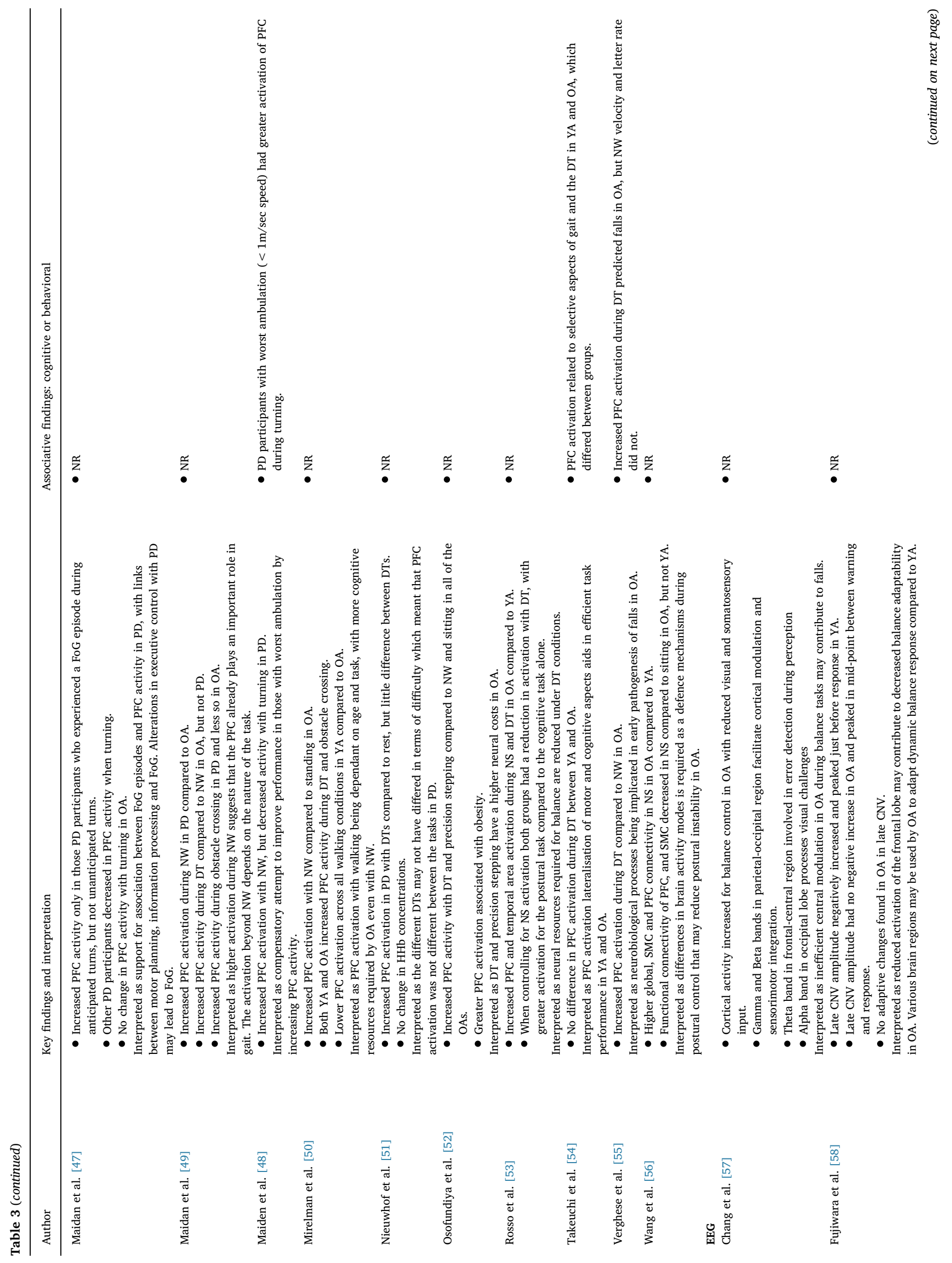




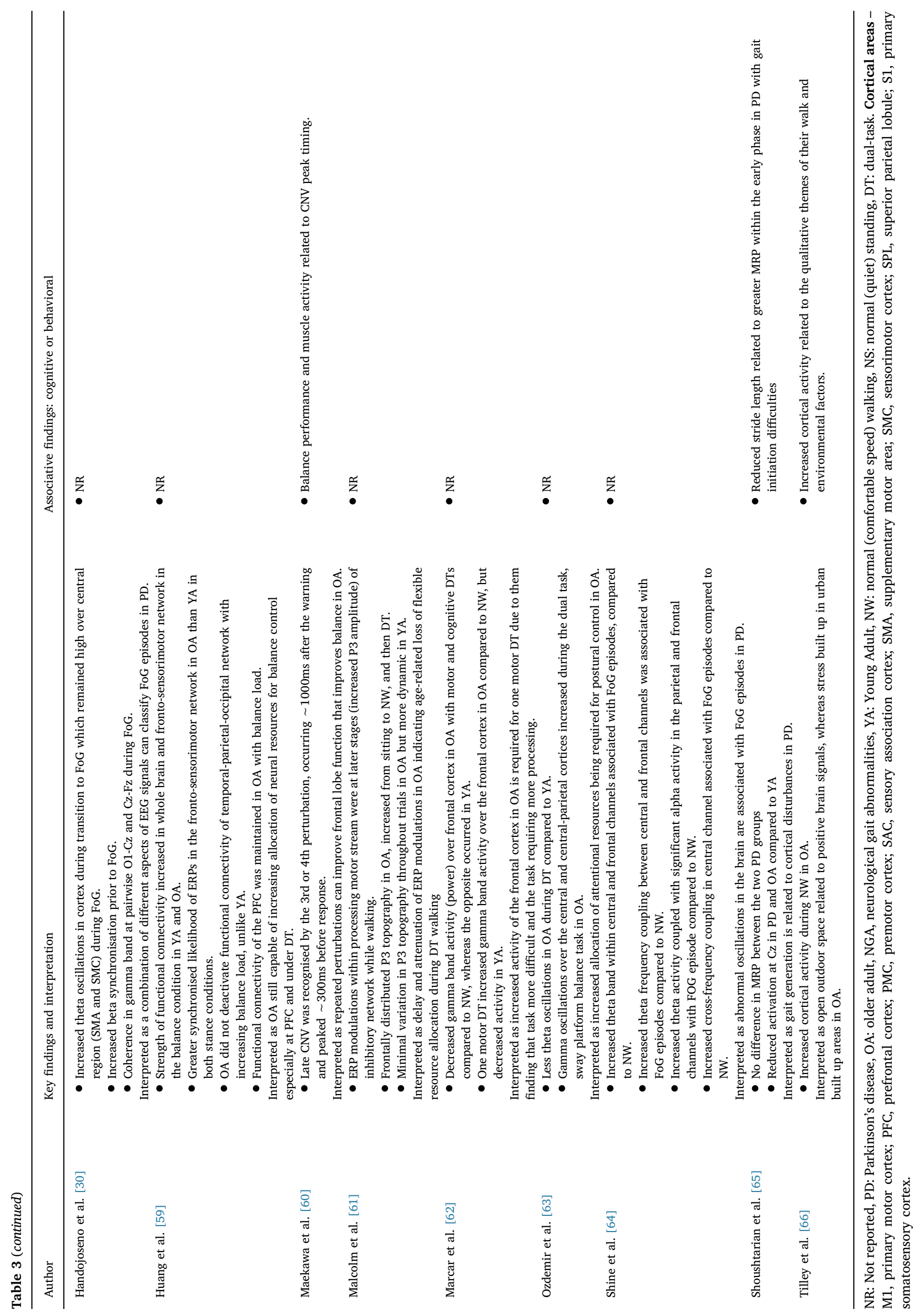




\section{Older Adults}

\section{Walking}

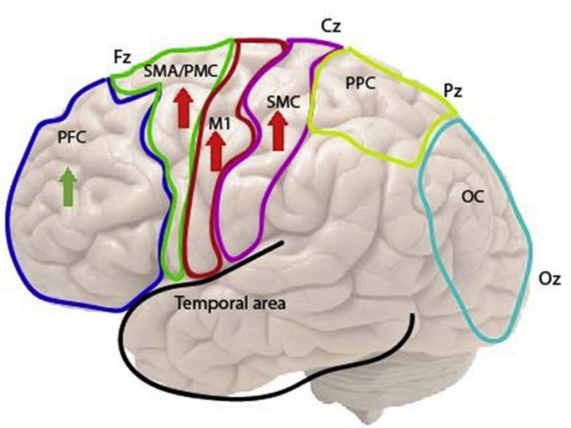

\section{Parkinson's disease}

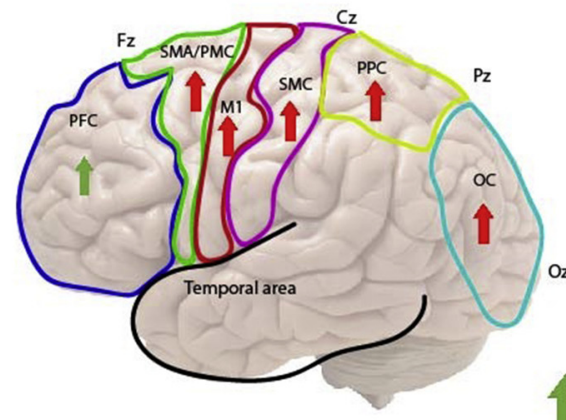

Strong evidence (Within >3 studies)
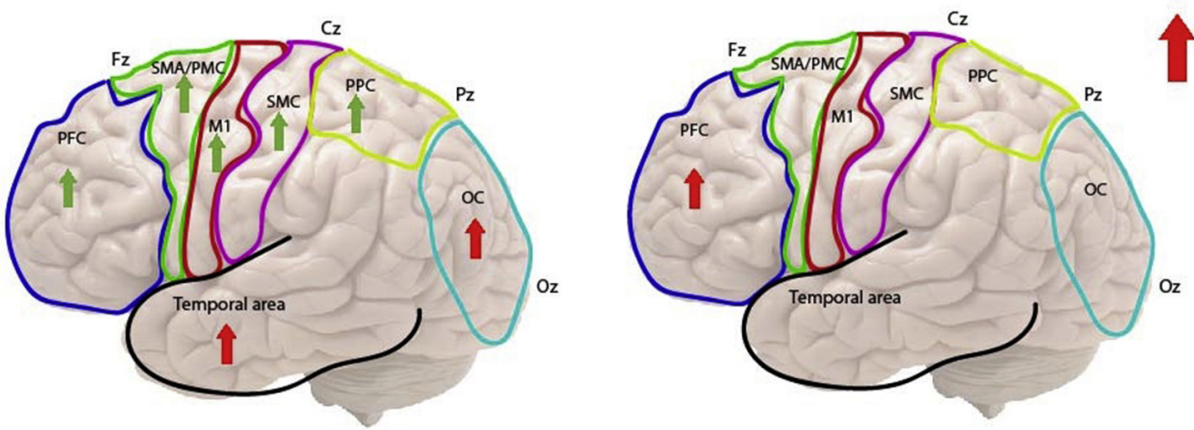

Weak evidence

(Within $<3$ studies)

\section{Balance}

Fig. 3. Overview of cortical activation recorded from fNIRS and EEG during walking and balance tasks in older adults and Parkinson's disease. [Arrows represent findings of increased cortical activity during the separate tasks within specific brain regions].

Timed-up-and-go or gait initiation) which is likely an attempt to avoid movement artefacts [68]. In contrast, fNIRS was used for freely moveable tasks (i.e. prolonged walking over-ground) as it is less prone to noise than EEG [69]. However, fNIRS has a mobility-resolution trade-off. Specifically, sampling frequencies are low and hemodynamic response can take 4-7s [70], which makes real-time examination difficult. Alternatively, EEG provides high-resolution sampling frequency that can examine the activation at multiple brain regions in real-time. However, devices capable of recording with minimal movement artefact have yet to be implemented in older adult and PD research [71-73]. Overall, there is no reported 'gold standard' device for monitoring cortical activity during walking or balance tasks.

\subsection{Regions of interest}

Various ROIs were investigated within older adult and PD participants during walking and balance tasks, which appeared to be based more upon technological limitations rather than task-specific regions. For example, the majority of the fNIRS studies examined the PFC, which is likely due to the headband nature of the devices not allowing examination of other ROIs and problems with wireless streaming of multiple channels from a single device. This limitation was further evident through one study using two fNIRS systems to collect from multiple ROIs [56]. Alternatively, EEG systems allowed multiple ROIs to be included. However, despite collecting data from a large array of EEG channels, studies tended to report data from few selected channels ( $n=3-4)$, thus limiting the interpretation of EEG results; some studies suggest a minimum of 35 channels is required for accurate mobile data reporting [74]. In addition, several studies examined data from the same channel (i.e. Cz) but attributed recordings to different ROIs, which further highlights the need for a standardized approach to ensure the correct interpretation of age- or PD-related deficits.

\subsection{Pre-processing and outcome measures}

Currently there are no 'gold standard' cortical activity outcomes or pre-processing of the signals, which was evident from the wide range of reported metrics and signal processing techniques, particularly within EEG studies. Unstandardized reporting impacted EEG outcome thresholds that likely explain inconsistent findings during the same task in PD $[30,64]$. Depending upon the outcome definition/threshold, valuable information may be discarded from, or irrelevant information included within, the analysis. Outcomes also did not appear to be reported in a task-specific manner, although this may be influenced by the software and analysis methods available. Although the majority of fNIRS studies reported the same outcome $\left(\mathrm{HbO}_{2}\right)$, they also recorded other metrics ( $\mathrm{HHb}$, TOI etc.) but did not present these results, limiting the interpretation. Creating optimal strategies for reporting fNIRS and EEG outcomes is difficult with different instruments and methods [75]. Therefore, studies should emphatically report outcomes and explain reasons for thresholds or focusing on particular outcomes. For example, EEG gamma band analysis may be a focus due to links with attentional processing $[76,77]$.

\subsection{Interpretation of outcomes}

The reviewed studies primarily examined older adults, with few PD specific studies finding that both ageing and PD consistently influenced cortical activity during walking and balance tasks. Cortical activity tended to increase with walking and balance tasks in both groups compared to baseline conditions (sitting/standing) or controls, particularly within frontal regions and their projected networks (Fig. 3). Greater cortical activity increases were required in PD, with attenuation of activation in older adults with repeated task exposure. Despite a lack of consistent secondary cognitive/motor task between studies, dualtasks exacerbated the increase in cortical activation, particularly in PD, which was suggested to be due to reduced neural resource availability in PD. Several studies attributed increased activation as executive- 
attentional compensation for either age- or PD-related motor control deficits $[41,45,48]$, which supports previous behavioral literature [15-17]. However, to establish this theory examination of relationships between cognitive functions and cortical activity are needed, as well as use of standardized non-spoken dual-task paradigms to avoid data collection issues [70]. Other studies have suggested that older adults may have reduced ability to selectively activate brain regions or mechanisms and integrate these with movement, which leads to over-recruitment of regions [78]. Overall, studies suggest that motor control becomes cortically mediated with age and further with PD pathology, which may be an attempt to bypass or compensate for dysfunctional sub-cortical automatic locomotor circuits.

Recruitment of cortical regions was also related to task demands, with specific walking tasks either increasing or decreasing activation of different ROIs. For example; turning had no effect and reduced PFC activity in older adults and PD, respectively. Whereas obstacle crossing or precision stepping increased PFC activity in older adults, and TUG or treadmill walking increased frontal and central cortical activity in PD. Correspondingly, complex motor tasks have been suggested to require cortical activation $[79,80]$ and animal models have demonstrated that activation depends on task specifics [81]. Within PD, activation also appeared to depend upon specific motor deficits. A fascinating intermittent phenomenon in PD is freezing of gait (FoG), which is a brief absence or reduction in walking that relates to mobility deficits and falls risk. Increased cortical activity was associated with FoG episodes during walking tasks, which supports theories regarding FoG being caused by increased cognitive task demand $[82,83]$, as well as bottleneck [84] and interference [85] theories of cognitive input into motor performance.

Unfortunately, few studies reported association between cortical outcomes and behavioral data (Table 3), which limits clinical interpretation. The few reported findings demonstrated that the ability to increase cortical activity related to reduced falls in older adults [55] and may improve with an exercise program [38], with direct clinical implication. Other studies highlighted that increased PFC activity related to worse gait (slower speed), gender, stress and fatigue in older adults $[43,44,46]$. Similarly, increased PFC activity during turning and greater MRP related to worse gait in PD $[48,65]$. Balance performance also related to cortical timing metrics in older adults [60]. However, cautious application of these findings to clinical practice is required due to the methodological limitations discussed.

\subsection{Clinical and future research recommendations}

Cortical activity appears to increase from baseline measures with walking and balance task performance in both older adults and PD, but more so in PD. This may represent cortical compensation for sub-cortical dysfunction with ageing and further with disease-pathology. Therefore, targeting cortical activation, particularly executive-attentional activity at the PFC, with interventions such as transcranial magnetic or direct current stimulation, pharma-logical or cueing strategies may help to alleviate the cortical burden of walking and balance tasks in PD. However, a lack of standardized approach to studying cortical activity during walking and balance tasks limits understanding and application to clinical practice. Therefore, we make the following recommendations for future research;

- Use adequately powered sample sizes

- Use task-appropriate instrumentation with an adequately justified sampling rate and number of channels for relevant outcomes

- Provide detailed information on ROIs and justify the number and location of channels assigned to each ROI

- Emphatically report all recorded cortical activity outcomes and provide outcome definitions along with a detailed report of data preprocessing

- Use standard walking (ideally overground) or balance tasks that can be compared to previous literature

- Use standard secondary tasks that do not interfere with cortical activity instrumentation measurement (i.e. no talking)

- Routinely assess relationships between cortical activity and behavioral/cognitive outcomes

\section{Conclusions}

In conclusion, cortical activity during walking and balance tasks was shown to be sensitive to age and PD, with overall increased activation required for task performance in both groups, but more so in PD. However, current results are limited due to a lack of standardized approach, which future studies must address to ensure accurate and appropriate data interpretation.

\section{Contributors}

Samuel Stuart developed the concept for the paper, gathered references, wrote each draft and formatted the manuscript to meet journal requirements.

Rodrigo Vitorio, Rosie Morris, Douglas N. Martini and Peter C. Fino checked and gathered references, and commented on and added to each draft.

Martina Mancini led on the research topic and commented on each draft.

\section{Conflict of interest}

The authors declare that they have no conflict of interest.

\section{Funding}

This work has been supported by grants from the National Institute of Health via Career Development Award5R00 HD078492-04 (PI Mancini) and a PCO Pilot Grant Award (PI Mancini). This research was also supported in part by Sao Paulo Research Foundation (FAPESP, grant \#2014/22308-0) as a postdoctoral fellowship to Rodrigo Vitorio.

\section{Provenance and peer review}

This article has undergone peer review.

\section{Acknowledgements}

The authors would like to thank Oregon Health and Science University for providing the facilities that allowed the search strategy for this review to be conducted.

\section{Appendix A. Supplementary data}

Supplementary data associated with this article can be found, in the online version, at https://doi.org/10.1016/j.maturitas.2018.04.011.

\section{References}

[1] B. Galna, S. Lord, D.J. Burn, L. Rochester, Progression of gait dysfunction in incident Parkinson's disease: impact of medication and phenotype, Mov. Disord. 30 (3) (2015) 359-367.

[2] B. Schoneburg, M. Mancini, F. Horak, J.G. Nutt, Framework for understanding balance dysfunction in Parkinson's disease, Mov. Disord. 28 (11) (2013) 1474-1482.

[3] M. Montero-Odasso, J. Verghese, O. Beauchet, J.M. Hausdorff, Gait and cognition: a complementary approach to understanding brain function and the risk of falling, $\mathrm{J}$. Am. Geriatr. Soc. 60 (11) (2012) 2127-2136.

[4] J. Verghese, A. LeValley, C.B. Hall, M.J. Katz, A.F. Ambrose, R.B. Lipton, Epidemiology of gait disorders in community-residing older adults, J. Am. Geriatr. Soc. 54 (2) (2006) 255-261.

[5] L.Z. Rubenstein, Falls in older people: epidemiology, risk factors and strategies for prevention, Age Aging 35 (Suppl. 2) (2006) ii37-ii41. 
[6] T. Gazibara, T. Pekmezovic, D. Kisic-Tepavcevic, M. Svetel, A. Tomic, I. Stankovic, V.S. Kostic, Incidence and prediction of falls in Parkinson's disease: a prospective cohort study, Eur. J. Epidemiol. 30 (4) (2015) 349-352.

[7] D.S. Peterson, F.B. Horak, Neural control of walking in people with Parkinsonism, Physiology (Bethesda Md.) 31 (2) (2016) 95-107.

[8] K. Takakusaki, Neurophysiology of gait: from the spinal cord to the frontal lobe, Mov. Disord. 28 (11) (2013) 1483-1491.

[9] K. Takakusaki, J. Oohinata-Sugimoto, K. Saitoh, T. Habaguchi, Role of basal ganglia-brainstem systems in the control of postural muscle tone and locomotion, Prog. Brain Res. 143 (2004) 231-237.

[10] T. Wu, M. Hallett, P. Chan, Motor automaticity in Parkinson's disease, Neurobiol. Dis. 82 (2015) 226-234.

[11] T. Hanakawa, Neuroimaging of standing and walking: special emphasis on Parkinsonian gait, Parkinsonism Relat. Disorders 12 (2006) S70-S75.

[12] D.J. Clark, Automaticity of walking: functional significance, mechanisms, measurement and rehabilitation strategies, Front. Hum. Neurosci. 9 (2015) 246.

[13] T. Wu, M. Hallett, A functional MRI study of automatic movements in patients with Parkinson's disease, Brain 128 (Pt 10) (2005) 2250-2259.

[14] N.I. Bohnen, K.A. Frey, S. Studenski, V. Kotagal, R.A. Koeppe, P.J. Scott, R.L. Albin, M.L. Müller, Gait speed in Parkinson disease correlates with cholinergic degeneration, Neurology 81 (18) (2013) 1611-1616.

[15] G. Yogev-Seligmann, J.M. Hausdorff, N. Giladi, The role of executive function and attention in gait, Mov. Disord. 23 (3) (2008) 329-342 (quiz 472).

[16] R. Morris, S. Lord, J. Bunce, D. Burn, L. Rochester, Gait and cognition. Mapping the global and discrete relationships in ageing and neurodegenerative disease, Neurosci. Biobehav. Rev. 64 (2016) 326-345.

[17] V.E. Kelly, A.J. Eusterbrock, A. Shumway-Cook, A review of dual-task walking deficits in people with Parkinson's disease: motor and cognitive contributions, mechanisms, and clinical implications, Parkinson's Disease 2012 (2012) 918719.

[18] R.D. Seidler, J.A. Bernard, T.B. Burutolu, B.W. Fling, M.T. Gordon, J.T. Gwin, Y. Kwak, D.B. Lipps, Motor control and aging links to age-related brain structural, functional, and biochemical effects, Neurosci. Biobehav. Rev. 34 (5) (2010) 721-733.

[19] Q. Tian, N. Chastan, W.-N. Bair, S.M. Resnick, L. Ferrucci, S.A. Studenski, The brain map of gait variability in aging, cognitive impairment and dementia-a systematic review, Neurosci. Biobehav. Rev. 74 (Part A) (2017) 149-162.

[20] F. Agosta, E. Canu, E. Stefanova, L. Sarro, A. Tomić, V. Špica, G. Comi, V.S. Kostić, M. Filippi, Mild cognitive impairment in Parkinson's disease is associated with a distributed pattern of brain white matter damage, Hum. Brain Mapp. 35 (5) (2014) 1921-1929.

[21] B. Sehm, M. Taubert, V. Conde, D. Weise, J. Classen, J. Dukart, B. Draganski, A. Villringer, P. Ragert, Structural brain plasticity in Parkinson's disease induced by balance training, Neurobiol. Aging 35 (1) (2014) 232-239.

[22] K. Rosenberg-Katz, T. Herman, Y. Jacob, E. Kliper, N. Giladi, J.M. Hausdorff, Subcortical volumes differ in Parkinson's disease motor subtypes: new insights into the pathophysiology of disparate symptoms, Front. Hum. Neurosci. 10 (2016).

[23] F.A. Sorond, Y. Cruz-Almeida, D.J. Clark, A. Viswanathan, C.R. Scherzer, P. De Jager, A. Csiszar, P.J. Laurienti, J.M. Hausdorff, W.G. Chen, L. Ferrucci, C. Rosano, S.A. Studenski, S.E. Black, L.A. Lipsitz, Aging, the central nervous system, and mobility in older adults: neural mechanisms of mobility impairment, J. Gerontol. Ser. A 70 (12) (2015) 1526-1532.

[24] R. Holtzer, N. Epstein, J.R. Mahoney, M. Izzetoglu, H.M. Blumen, Neuroimaging of mobility in aging: a targeted review, J. Gerontol. Ser. A: Biol. Sci. Med. Sci. 69 (11) (2014) 1375-1388.

[25] A. Zwergal, J. Linn, G. Xiong, T. Brandt, M. Strupp, K. Jahn, Aging of human supraspinal locomotor and postural control in fMRI, Neurobiol. Aging 33 (6) (2012) 1073-1084.

[26] M. Bakker, F.P. De Lange, R.C. Helmich, R. Scheeringa, B.R. Bloem, I. Toni, Cerebral correlates of motor imagery of normal and precision gait, Neuroimage 41 (3) (2008) 998-1010.

[27] D. Hamacher, F. Herold, P. Wiegel, D. Hamacher, L. Schega, Brain activity during walking: a systematic review, Neurosci. Biobehav. Rev, 57 (2015) 310-327.

[28] C. la Fougere, A. Zwergal, A. Rominger, S. Forster, G. Fesl, M. Dieterich, T. Brandt, M. Strupp, P. Bartenstein, K. Jahn, Real versus imagined locomotion: a [18F]-FDG PET-fMRI comparison, Neuroimage 50 (4) (2010) 1589-1598.

[29] D. Moher, A. Liberati, J. Tetzlaff, D.G. Altman, Preferred reporting items for systematic reviews and meta-analyses: the PRISMA statement, PLoS Med. 6 (7) (2009) e1000097.

[30] A.M. Handojoseno, J.M. Shine, T.N. Nguyen, Y. Tran, S.J.G. Lewis, H.T. Nguyen, Analysis and prediction of the freezing of gait using EEG brain dynamics, IEEE Trans. Neural Syst. Rehabil. Eng. 23 (5) (2015) 887-896.

[31] J.R. Mahoney, R. Holtzer, M. Izzetoglu, V. Zemon, J. Verghese, G. Allali, The role of prefrontal cortex during postural control in Parkinsonian syndromes a functional near-infrared spectroscopy study, Brain Res. 1633 (2016) 126-138.

[32] R. Beurskens, I. Helmich, R. Rein, O. Bock, Age-related changes in prefrontal activity during walking in dual-task situations: a fNIRS study, Int. J. Psychophysiol. 92 (3) (2014) 122-128.

[33] G. Chaparro, J.M. Balto, B.M. Sandroff, R. Holtzer, M. Izzetoglu, R.W. Motl, M.E. Hernandez, Frontal brain activation changes due to dual-tasking under partial body weight support conditions in older adults with multiple sclerosis, J. Neuroeng. Rehabil. 14 (1) (2017).

[34] M. Chen, S. Pillemer, S. England, M. Izzetoglu, J.R. Mahoney, R. Holtzer, Neural correlates of obstacle negotiation in older adults: an fNIRS study, Gait Posture 58 (2017) 130-135.

[35] D.J. Clark, E.A. Christou, S.A. Ring, J.B. Williamson, L. Doty, Enhanced somatosensory feedback reduces prefrontal cortical activity during walking in older adults,
J. Gerontol. Ser. A Biol. Sci. Med. Sci. 69 (11) (2014) 1422-1428.

[36] D.J. Clark, D.K. Rose, S.A. Ring, E.C. Porges, Utilization of central nervous system resources for preparation and performance of complex walking tasks in older adults, Front. Aging Neurosci. 6 (August) (2014).

[37] T. Doi, H. Makizako, H. Shimada, H. Park, K. Tsutsumimoto, K. Uemura, T. Suzuki, Brain activation during dual-task walking and executive function among older adults with mild cognitive impairment: a fNIRS study, Aging Clin. Exp. Res. 25 (5) (2013) 539-544.

[38] P. Eggenberger, M. Wolf, M. Schumann, E.D. de Bruin, Exergame and balance training modulate prefrontal brain activity during walking and enhance executive function in older adults, Front. Aging Neurosci. 8 (April) (2016).

[39] S.A. Fraser, O. Dupuy, P. Pouliot, F. Lesage, L. Bherer, Comparable cerebral oxygenation patterns in younger and older adults during dual-task walking with increasing load, Front. Aging Neurosci. 8 (October) (2016).

[40] T. Harada, I. Miyai, M. Suzuki, K. Kubota, Gait capacity affects cortical activation patterns related to speed control in the elderly, Exp. Brain Res. 193 (3) (2009) $445-454$.

[41] M.E. Hernandez, R. Holtzer, G. Chaparro, K. Jean, J.M. Balto, B.M. Sandroff, M. Izzetoglu, R.W. Motl, Brain activation changes during locomotion in middleaged to older adults with multiple sclerosis, J. Neurol. Sci. 370 (2016) 277-283.

[42] R. Holtzer, J.R. Mahoney, M. Izzetoglu, K. Izzetoglu, B. Onaral, J. Verghese, fNIRS study of walking and walking while talking in young and old individuals, J. Gerontol. Ser. A Biol. Sci. Med. Sci. 66 A (8) (2011) 879-887.

[43] R. Holtzer, J.R. Mahoney, M. Izzetoglu, C. Wang, S. England, J. Verghese, Online fronto-cortical control of simple and attention-demanding locomotion in humans, Neuroimage 112 (2015) 152-159.

[44] R. Holtzer, C. Schoen, E. Demetriou, J.R. Mahoney, M. Izzetoglu, C. Wang, J. Verghese, Stress and gender effects on prefrontal cortex oxygenation levels assessed during single and dual-task walking conditions, Eur. J. Neurosci. 45 (5) (2017) 660-670.

[45] R. Holtzer, J. Verghese, G. Allali, M. Izzetoglu, C. Wang, J.R. Mahoney, Neurological gait abnormalities moderate the functional brain signature of the posture first hypothesis, Brain Topogr. 29 (2) (2016) 334-343.

[46] R. Holtzer, J. Yuan, J. Verghese, J.R. Mahoney, M. Izzetoglu, C. Wang, Interactions of subjective and objective measures of fatigue defined in the context of brain control of locomotion, the journals of gerontology, Ser. A, Biol. Sci. Med. Sci. 72 (3) (2017) 417-423.

[47] I. Maidan, H. Bernad-Elazari, E. Gazit, N. Giladi, J.M. Hausdorff, A. Mirelman, Changes in oxygenated hemoglobin link freezing of gait to frontal activation in patients with Parkinson disease: an fNIRS study of transient motor-cognitive fail ures, J. Neurol. 262 (4) (2015) 899-908.

[48] I. Maidan, H. Bernad-Elazari, N. Giladi, J.M. Hausdorff, A. Mirelman, When is higher level cognitive control needed for locomotor tasks among patients with Parkinson's disease? Brain Topogr. (2017) 1-8.

[49] I. Maidan, F. Nieuwhof, H. Bernad-Elazari, M.F. Reelick, B.R. Bloem, N. Giladi, J.E. Deutsch, J.M. Hausdorff, J.A. Claassen, A. Mirelman, The role of the frontal lobe in complex walking among patients with Parkinson's disease and healthy older adults: an fNIRS study, Neurorehabil. Neural Repair 30 (10) (2016) 963-971.

[50] A. Mirelman, I. Maidan, H. Bernad-Elazari, S. Shustack, N. Giladi, J.M. Hausdorff, Effects of aging on prefrontal brain activation during challenging walking conditions, Brain Cogn. 115 (2017) 41-46.

[51] F. Nieuwhof, M.F. Reelick, I. Maidan, A. Mirelman, J.M. Hausdorff, M.G.O. Rikkert, B.R. Bloem, M. Muthalib, J.A. Claassen, Measuring prefrontal cortical activity during dual task walking in patients with Parkinson's disease: feasibility of using a new portable fNIRS device, Pilot Feasibil. Stud. 2 (1) (2016) 59.

[52] O. Osofundiya, M.E. Benden, D. Dowdy, R.K. Mehta, Obesity-specific neural cost of maintaining gait performance under complex conditions in community-dwelling older adults, Clin. Biomech. 35 (2016) 42-48.

[53] A.L. Rosso, M. Cenciarini, P.J. Sparto, P.J. Loughlin, J.M. Furman, T.J. Huppert, Neuroimaging of an attention demanding dual-task during dynamic postural control, Gait Posture 57 (2017) 193-198.

[54] N. Takeuchi, T. Mori, Y. Suzukamo, N. Tanaka, S.I. Izumi, Parallel processing of cognitive and physical demands in left and right prefrontal cortices during smartphone use while walking, BMC Neurosci. 17 (1) (2016).

[55] J. Verghese, C. Wang, E. Ayers, M. Izzetoglu, R. Holtzer, Brain activation in highfunctioning older adults and falls: prospective cohort study, Neurology 88 (2) (2017) 191-197.

[56] B. Wang, M. Zhang, L. Bu, L. Xu, W. Wang, Z. Li, Posture-related changes in brain functional connectivity as assessed by wavelet phase coherence of NIRS signals in elderly subjects, Behav. Brain Res. 312 (2016) 238-245.

[57] C.-J. Chang, T.-F. Yang, S.-W. Yang, J.-S. Chern, Cortical modulation of motor control biofeedback among the elderly with high fall risk during a posture perturbation task with augmented reality, Front. Aging Neurosci. 8 (2016) 80.

[58] K. Fujiwara, M. Maekawa, N. Kiyota, C. Yaguchi, Adaptation changes in dynamic postural control and contingent negative variation during backward disturbance by transient floor translation in the elderly, J. Physiol. Anthropol. 31 (2012) 12.

[59] C.Y. Huang, L.L. Lin, I.S. Hwang, Age-related differences in reorganization of functional connectivity for a dual task with increasing postural destabilization, Front. Aging Neurosci. 9 (April) (2017).

[60] M. Maekawa, K. Fujiwara, N. Kiyota, C. Yaguchi, Adaptation changes in dynamic postural control and contingent negative variation during repeated transient forward translation in the elderly, J. Physiol. Anthropol. 32 (1) (2013).

[61] B.R. Malcolm, J.J. Foxe, J.S. Butler, P. De Sanctis, The aging brain shows less flexible reallocation of cognitive resources during dual-task walking: a mobile brain/body imaging (MoBI) study, Neuroimage 117 (2015) 230-242.

[62] V.L. Marcar, S.A. Bridenbaugh, J. Kool, K. Niedermann, R.W. Kressig, A simple 
procedure to synchronize concurrent measurements of gait and brain electrical activity and preliminary results from a pilot measurement involving motor-cognitive dual-tasking in healthy older and young volunteers, J. Neurosci. Methods 228 (2014) 46-49.

[63] R.A. Ozdemir, J.L. Contreras-Vidal, B.C. Lee, W.H. Paloski, Cortical activity modulations underlying age-related performance differences during posture-cognition dual tasking, Exp. Brain Res. 234 (11) (2016) 3321-3334.

[64] J.M. Shine, A.M.A. Handojoseno, T.N. Nguyen, Y. Tran, S.L. Naismith, H. Nguyen, S.J.G. Lewis, Abnormal patterns of theta frequency oscillations during the temporal evolution of freezing of gait in parkinson's disease, Clin. Neurophysiol. 125 (3) (2014) 569-576

[65] M. Shoushtarian, A. Murphy, R. Iansek, Examination of central gait control mechanisms in Parkinson's disease using movement-related potentials, Mov. Disord. 26 (13) (2011) 2347-2353.

[66] S. Tilley, C. Neale, A. Patuano, S. Cinderby, Older people's experiences of mobility and mood in an urban environment: a mixed methods approach using electroencephalography (EEG) and interviews, Int. J. Environ. Res. Public Health 14 (2) (2017).

[67] M.M. Plichta, M.J. Herrmann, C.G. Baehne, A.C. Ehlis, M.M. Richter, P. Pauli, A.J. Fallgatter, Event-related functional near-infrared spectroscopy (fNIRS): Are the measurements reliable? Neuroimage 31 (1) (2006) 116-124.

[68] J.E. Kline, H.J. Huang, K.L. Snyder, D.P. Ferris, Isolating gait-related movement artifacts in electroencephalography during human walking, J. Neural Eng. 12 (4) (2015) 046022-046022.

[69] R.J. Cooper, J. Selb, L. Gagnon, D. Phillip, H.W. Schytz, H.K. Iversen, M. Ashina, D.A. Boas, A systematic comparison of motion artifact correction techniques for functional near-infrared spectroscopy, Front. Neurosci. 6 (2012).

[70] R. Vitorio, S. Stuart, L. Rochester, L. Alcock, A. Pantall, fNIRS response during walking - artefact or cortical activity? A systematic review, Neurosci. Biobehav. Rev. 83 (2017) 160-172.

[71] K. Nathan, J.L. Contreras-Vidal, Negligible motion artifacts in scalp electroencephalography (EEG) during treadmill walking, Front. Hum. Neurosci. 9 (708) (2016)

[72] A. Melnik, P. Legkov, K. Izdebski, S.M. Kärcher, W.D. Hairston, D.P. Ferris, P. König, Systems, subjects, sessions to what extent do these factors influence EEG data?
Front. Hum. Neurosci. 11 (150) (2017).

[73] A.S. Oliveira, B.R. Schlink, W.D. Hairston, P. Konig, D.P. Ferris, Induction and separation of motion artifacts in EEG data using a mobile phantom head device, J. Neural Eng. 13 (3) (2016) 036014

[74] T.M. Lau, J.T. Gwin, D.P. Ferris, How many electrodes are really needed for EEGbased mobile brain imaging? J. Behav. Brain Sci. 2 (3) (2012) 4.

[75] A.S. Oliveira, B.R. Schlink, W.D. Hairston, P. König, D.P. Ferris, Proposing metrics for benchmarking novel EEG technologies towards real-world measurements, Front. Hum. Neurosci. 10 (188) (2016).

[76] S. Slobounov, C. Cao, N. Jaiswal, K.M. Newell, Neural basis of postural instability identified by VTC and EEG: experimental brain research, Experimentelle Hirnforschung. Exp. Cereb. 199 (1) (2009) 1-16.

[77] S. Slobounov, W. Sebastianelli, R. Moss, Alteration of posture-related cortical potentials in mild traumatic brain injury, Neurosci. Lett. 383 (3) (2005) 251-255.

[78] J.M. Logan, A.L. Sanders, A.Z. Snyder, J.C. Morris, R.L. Buckner, Under-recruitment and nonselective recruitment: dissociable neural mechanisms associated with aging, Neuron 33 (5) (2002) 827-840.

[79] T. Verstynen, J. Diedrichsen, N. Albert, P. Aparicio, R.B. Ivry, Ipsilateral motor cortex activity during unimanual hand movements relates to task complexity, $\mathrm{J}$. Neurophysiol. 93 (3) (2005) 1209-1222.

[80] T.S. Braver, J.D. Cohen, L.E. Nystrom, J. Jonides, E.E. Smith, D.C. Noll, A parametric study of prefrontal cortex involvement in human working memory, Neuroimage 5 (1) (1997) 49-62.

[81] W.F. Asaad, G. Rainer, E.K. Miller, Task-specific neural activity in the primate prefrontal cortex, J. Neurophysiol. 84 (1) (2000) 451-459.

[82] S.J.G. Lewis, R.A. Barker, A pathophysiological model of freezing of gait in Parkinson's disease, Parkinsonism Relat. Disorders 15 (5) (2009) 333-338.

[83] J. Vandenbossche, N. Deroost, E. Soetens, J. Spildooren, S. Vercruysse, A. Nieuwboer, E. Kerckhofs, Freezing of gait in Parkinson disease is associated with impaired conflict resolution, Neurorehabil. Neural Repair 25 (8) (2011) 765-773.

[84] M. Sigman, S. Dehaene, Dynamics of the central bottleneck: dual-task and task uncertainty, PLoS Biol. 4 (7) (2006) e220.

[85] H. Pashler, Dual-task interference in simple tasks: data and theory, Psychol. Bull. 116 (2) (1994) 220 